\title{
Effects of External Magnetic Field and Air Mass on Space Charge Region Width Extension of a Bifacial Solar Cell Front Side Illumination
}

\author{
Alain Diasso ${ }^{1, *}$, Raguilignaba Sam ${ }^{1,2}$, Nazé Yacouba Traoré2 François Zougmoré $^{1}$ \\ ${ }^{1}$ Laboratoire de Matériaux et Environnement, UFR/SEA, Université Joseph Ki-Zerbo, Ouagadougou, Burkina Faso \\ ${ }^{2}$ Departement de Physique, UFR/ST, Université Nazi Boni, Bobo Dioulasso, Burkina Faso
}

Email address:

alinodiass@yahoo.fr (A. Diasso),samrsedou@yahoo.fr (R. Sam),nazeyacouba@yahoo.com (N. Y. Traoré),

fr.zougmore@laposte.net (F. Zougmoré)

${ }^{*}$ Corresponding author

\section{To cite this article:}

Alain Diasso, Raguilignaba Sam, Nazé Yacouba Traoré, François Zougmoré. Effects of External Magnetic Field and Air Mass on Space Charge Region Width Extension of a Bifacial Solar Cell Front Side Illumination. International Journal of Energy and Power Engineering. Vol. 9, No. 3, 2020, pp. 29-34. doi: 10.11648/j.ijepe.20200903.11

Received: June 29, 2020; Accepted: July 29, 2020; Published: July 30, 2020

\begin{abstract}
The environmental and economical merits of converting solar energy into electricity via photovoltaic cells have caused an ever increasing interest among developed and developing countries to allocate more budget on photovoltaic systems in order to boost up their efficiency in recent years. Besides the material and design parameters, there are several external factors such as magnetic field, air mass, intense light, external electric field, solar spectrum.... that can influence the PV cell's performance. There have been a handful of studies conducted on the effect of various influential parameters on the efficiency and performance of photovoltaic cells; however none has taken these two parameters (magnetic field and air mass) into account simultaneously. In this 3D study the effects of magnetic field and the air mass illumination on space charge region width extension of a bifacial polycristalline solar cell front side illumination will be elaborated. Based on the columnar model of the grain and the quasi-neutral base, the continuity equation is established and the boundaries conditions are defined in order to use Green's functions to solve this equation. New analytical expression of charge carriers' density is found and the diffusion capacitance to the junction is calculated. The normalized carriers' density plot versus base depth and magnetic field with various air mass illumination are presented and analyzed. The effects of magnetic field and air mass illumination on space charge region width extension are then deducted. The influences of magnetic field and air mass illumination on the junction capacitance and on the reverse of junction capacitance are also shown and analyzed.
\end{abstract}

Keywords: Air Mass, Bifacial, Magnetic Field, Junction Capacitance, Space Charge Region

\section{Introduction}

The characterization of the performance of the solar cell do not take into account the effects of such environmental factors as insulation level, solar spectrum, electric field, magnetic field and other meteorological conditions. However, some external factors as magnetic field, external electric field, intense light, air mass and internal factors as grain size, grains boundaries recombination velocity, electric field in the bulk due to carrier concentration [1-12] can modify the solar cells operating whatever the solar cell quality.

Due to natural spectral sensitivity of solar cell devices, the solar spectrum (air mass) and magnetic field are two of those factors which may strongly influence the solar cell performance.

Whatever is solar cell quality, it is important at its installation moment to take into account an outside factors susceptible to lower efficiency: the magnetic field.

Since the parameters as charge carriers density, junction capacitance and reverse of junction capacitance of the solar cell are closely dependent of carriers' distribution in the cell, the magnetic field and air mass illumination will influence these parameters.

In this work, we present the effects of magnetic field and air mass illumination in 3D study of bifacial solar on space charge 
width extension and junction capacity.

\section{Theoretical Backgrounds}

\subsection{Study Assumptions}

This study is based on a 3D modeling of a bifacial polycrystalline silicon solar cell; we made the following assumptions:

a) Considering the weak thickness of the emitter and the space charge region, we neglected their contributions to the photocurrent, so the quasi-totality of the current is provided therefore by the base $[13,14]$;

b) The grains are under shape parallelepipedic $(2 \mathrm{a} ; 2 \mathrm{~b} ; \mathrm{H})$ and the joints of grains are perpendicular to the junction; 1. The surfaces between two adjacent grains and perpendicular to the junction are characterized by the same carriers recombination processes evaluated by a grain boundary recombination velocity $S g x=S g y=S g$

2. The electric field of crystal lattice is negligible [15];

3. Only the contribution of the base in the processes of generation is considered;

c) The magnetic field is oriented according to the direction $\overrightarrow{o y}, \vec{B}=\overrightarrow{B o y}$. It is therefore perpendicular to the depth of light penetration in order to observe the effect of the strength of Lorentz on the charge carriers;

d) The illumination is uniform. Then we have a generation rate depending only on the depth in the base z;

e) The solar cell is front side illuminated under the external magnetic field with various air mass illumination on the figure $1[16]$;

f) The insulation level number (sun number) $n=1$.

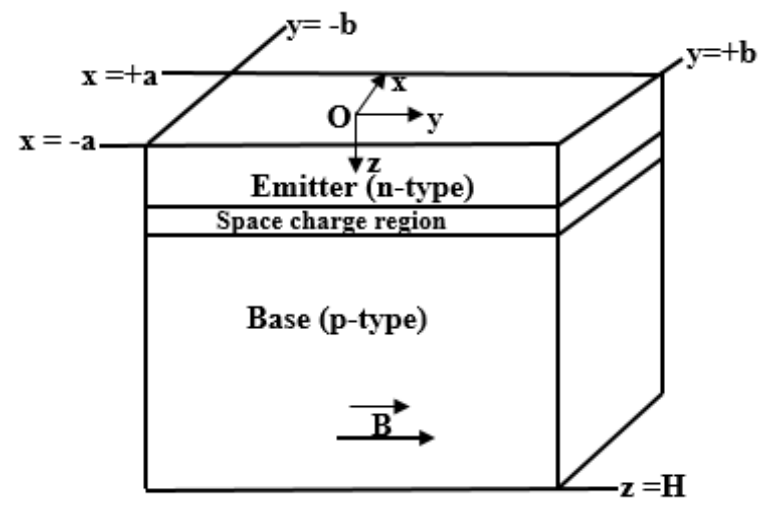

Figure 1. Theoretical model of a grain with an external magnetic field.

\subsection{Diffusion Equation Resolution}

In the base of the solar cell, the minority carriers are electrons, and their density satisfies to the equation below:

$$
\frac{\partial \delta n}{\partial t}=\frac{1}{e} \vec{\nabla} \cdot \vec{j}_{n}+g_{n}-r_{n}
$$

$g_{n}, r_{n}$ are respectively the rate of generation and recombination of carriers. The current density $\vec{j}_{n}$ is given by:

$$
\vec{j}_{n}=e D_{n} \vec{\nabla} \delta_{n}+e \mu_{n} \vec{E}-\mu_{n} \vec{j}_{n} \Lambda \vec{B}
$$

$\vec{E}$ is the electric field of the crystal lattice and $\mu_{n}$ is the mobility of the electrons.

Substituting equation (2) into (1) and taking into account the assumption of quasi-neutrality of the base, the diffusion equation of the electrons in the base becomes the equation:

$$
\frac{\partial \delta(\vec{r}, t)}{\partial t}-D^{*}\left(\vec{\nabla} \delta(\vec{r}, t)-\frac{\delta(\vec{r}, t)}{L^{*}}\right)=g(z, t)
$$

where $\delta(\vec{r}, t)=\delta(x, y, z, t)$

$D^{*}$ is the diffusion coefficient of excess minority carriers in the base of the bifacial cell in presence of magnetic field [18, 19]; it can be expressed as:

$$
D^{*}=\frac{D}{\theta}
$$

and

$$
\theta=1+(\mu B)^{2}
$$

$D, \mu, B$ are respectively the coefficient of electrons diffusion, the mobility of electrons and the value of magnetic field applied.

The presence of the magnetic field in our model lead to new values of carriers diffusion length $L^{*}$ and carriers diffusion coefficient $D^{*}$ which depend on magnetic field.

$\theta$ is a coefficient which depend on magnetic field intensity.

The carriers' generation rate under multispectral light at the depth $\mathrm{z}$ in the base can be written by the following expression:

$$
g(z, t)= \begin{cases}n \cdot \sum_{n=1}^{3} a_{m} \exp \left(-b_{m} z\right) & \text { si } 0 \leq \mathrm{t} \leq \mathrm{Te} \\ 0 & \text { si } t \succ T e\end{cases}
$$

In this expression of $g(z, t), n=\frac{I_{c c i}}{I_{c c o}}$ indicates the illumination level (sun number) and $n=1$ for our study.

The coefficients $a_{m}$ and $b_{m}$ are the modeling coefficients of illumination air mass values indicated on the table 1 [17, $18]$.

Table 1. Air mass illuminations values.

\begin{tabular}{lll}
\hline & $\mathrm{a}_{1}=16.10^{20}$ & $\mathrm{~b}_{1}=1,6.10^{4}$ \\
AM0 & $\mathrm{a}_{2}=2,25.10^{20}$ & $\mathrm{~b}_{2}=0,16.10^{4}$ \\
& $\mathrm{a}_{3}=0,085.10^{20}$ & $\mathrm{~b}_{3}=0,013.10^{4}$ \\
& $\mathrm{a}_{1}=12,3.10^{20}$ & $\mathrm{~b}_{1}=1,6.10^{4}$ \\
AM1 & $\mathrm{a}_{2}=0,54.10^{20}$ & $\mathrm{~b}_{2}=0,16.10^{4}$ \\
& $\mathrm{a}_{3}=0,0991.10^{20}$ & $\mathrm{~b}_{3}=0,013.10^{4}$ \\
& $\mathrm{a}_{1}=6,13.10^{20}$ & $\mathrm{~b}_{1}=6630$ \\
AM1.5 & $\mathrm{a}_{2}=0,54.10^{20}$ & $\mathrm{~b}_{2}=1000$ \\
& $\mathrm{a}_{3}=0,0991.10^{20}$ & $\mathrm{~b}_{3}=130$ \\
\hline
\end{tabular}


Equation (1) is solved with the following boundaries conditions:

At the junction $\mathrm{z}=0$

$$
\left.\frac{\partial \delta(\vec{r}, t)}{\partial z}\right|_{z=0}=\frac{S f}{D^{*}} \delta(\vec{r}(z=0), t)
$$

At the rear side $\mathrm{z}=\mathrm{H}$

$$
\left.\frac{\partial \delta(\vec{r}, t)}{\partial z}\right|_{z=H}=\frac{S b}{D^{*}} \delta(\vec{r}(z=H), t)
$$

At surfaces limited by $x= \pm a$ and $y= \pm b$

$$
\begin{gathered}
\left.\frac{\partial \delta(\vec{r}, t)}{\partial x}\right|_{x= \pm a}= \pm \frac{S g x}{D^{*}} \delta(\vec{r}(x= \pm a), t) \\
\left.\frac{\partial \delta(\vec{r}, t)}{\partial y}\right|_{y= \pm a}= \pm \frac{S g y}{D^{*}} \delta(\vec{r}(y= \pm b), t)
\end{gathered}
$$

$S f, S b$ and $S g$ are the recombination velocity of minority carriers respectively at surfaces $z=0, z=\mathrm{H}$ and $x= \pm$ $a($ or $y= \pm b)$.

$a, b$ and $H$ are the grain sizes as indicated on figure 1 .
A solution of equation (1) is given by expression (11) according to the author of the reference [16].

$$
G=\sum_{i=1}^{+\infty} \sum_{j=1}^{+\infty} \sum_{k=1}^{+\infty} M_{i} M_{j} M_{k} \exp \left(-\beta\left(t-t^{\prime}\right)\right)
$$

where:

$$
\begin{gathered}
M_{i}=A_{k_{i}}^{2} \cos \left(k_{i} x^{\prime}\right) \cos \left(k_{i} x\right) \\
M_{j}=A_{l_{j}}^{2} \cos \left(l_{j}^{*} y^{\prime}\right) \cos \left(l_{j}^{*} y\right) \\
M_{k}=A_{\mu_{k}}^{2} \cos \left(\mu_{k} z^{\prime}+\varphi_{k}\right) \cos \left(\mu_{k} z+\varphi_{k}\right)
\end{gathered}
$$

$\beta^{*}$ and $l_{j}^{*}$ are expressed by:

$$
\begin{gathered}
\beta^{*}=D^{*}\left(k^{2}{ }_{i}+l_{j}^{2}+\mu_{k}^{2}+\frac{1}{L^{* 2}}\right) \\
l_{j}^{*}=\frac{l_{j}}{\sqrt{\theta}}
\end{gathered}
$$

The expression (11) can be simply write for front side illumination:

$$
\delta_{1}(x, y, z, t)=\left[\frac{4 \cdot n \cdot a_{m} \cdot A_{k_{i, 1}}^{2} \cdot A_{l_{j, 1}}^{2} \cdot A_{\mu_{k, 1}}^{2} \times \sin \left(k_{i, 1} a\right) \sin \left(\frac{l_{j, 1}}{\sqrt{\theta}} b\right)}{k_{i, 1} \frac{l_{j, 1}}{\sqrt{\theta}} \beta^{*}} K \times \cos \left(k_{i, 1} \cdot x\right) \cdot \cos \left(\frac{l_{j, 1}}{\sqrt{\theta}} y\right) \times \cos \left(\mu_{k, 1} z+\varphi_{k, 1}\right)\right] \cdot F_{0}(t)
$$

where:

$$
\begin{gathered}
K=\frac{a_{m} \cdot b_{m}}{b_{m}{ }^{2}+\mu_{k, 1}^{2}}\left[-\exp \left(-b_{m} \cdot H\right) \cos \left(\mu_{k, 1} \cdot H+\varphi_{k, 1}\right)+\cos \left(\varphi_{k, 1}\right)+\frac{\mu_{k, 1}}{b_{m}} \exp \left(b_{m} \cdot H\right) \times \sin \left(\mu_{k, 1} \cdot H+\varphi_{k, 1}\right)-\frac{\mu_{k, 1}}{b_{m}} \sin \left(\varphi_{k, 1}\right)\right] \\
F_{0}(t)=\left(1-\exp \left(-\beta^{*} T e\right)\right) \cdot \exp \left(-\beta^{*}(t-T e)\right.
\end{gathered}
$$

The quantities $A_{l_{i}}, A_{l_{j}}$ and $A_{\mu_{k}}$ are obtained by normalizing $M_{i}, M_{j}$ and $M_{k}$.

The parameters $k_{i, 1}, l_{j, 1}$ and $\mu_{k, 1}$ are the eigenvalues obtained from the boundary conditions when solar cell is front side illumination.

$\varphi_{k, 1}$ is the initial phase and obtained by solving the equation

$$
\tan \left(\mu_{k, 1} H+\varphi_{k, 1}\right)=\frac{S b}{\mu_{k, 1} D^{*}}
$$

\section{Results and Discussions}

We deal here the simulation results obtained from the previous modeling equations from software Mathcad 15 and origin pro8.

\subsection{Effects of Air Mass on Charge Carriers Density}

Figure 2 present curves of carriers' density versus air mass and base depth $z$.

On this figure, we notice tree types of region whatever the curve:

1. Firstly, a region where the charge carriers density gradient is positive. These explain the passage of a flow of electrons through the junction;

2. Secondly, a region where the charge carriers density gradient is negative. These is translated by a gradual decrease in the incident light flux in the base depth thus reducing the carriers charge generation which contribute to the photocurrent;

3. Thirdly, a region where the charge carriers density gradient is zero. This region was limited by the two regions to the depth $z=z_{0}$. There is a storage of 
electrons which induce a capacity with a space charge region establishing itself to the junction $(z=0)$ to the base depth $z_{0}$.

In additional, we also note that the charge carriers density increase when the air mass of solar cell illumination decreases: we are therefore witnessing a reduction in charge carriers photogenerated in the base when the flow of incident light deviates from the perpendicular to the junction. Hence an increase in junction capacitance when the air mass of solar cell illumination increase.

So the space charge width extension has affected by the solar illumination air mass variations.

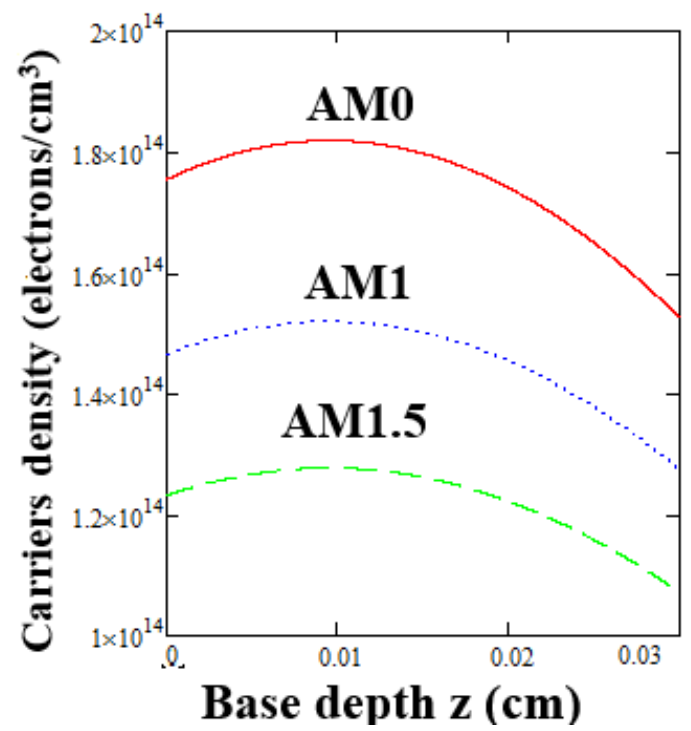

Figure 2. Carriers density profiles versus base depth with various air mass illumination values. $H=200 \mu \mathrm{m} ; S f=3.10^{3} \mathrm{~cm} / \mathrm{s}, S_{b}=200 \mathrm{~cm} / \mathrm{s}$.

\subsection{Effects of Magnetic Field on Charge Carriers Density}

The figure 3 shows the effects of magnetic field on charge carriers density.

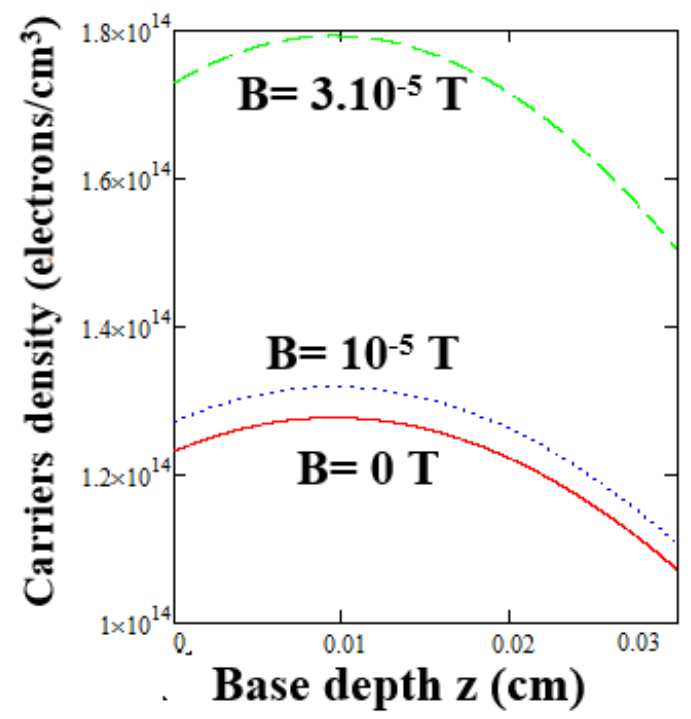

Figure 3. Carriers density profiles versus base depth with various magnetic field values under AM1.5. $H=200 \mu \mathrm{m} ; S f=3.10^{3} \mathrm{~cm} / \mathrm{s}, S_{b}=200 \mathrm{~cm} / \mathrm{s}$.
We remark tree forms of gradients whatever the curve:

1. The positive gradient $z \prec z_{0}$ : in this region the charge carriers cross the junction because they have more excess energy to cross junction and participate to current production

2. The zero gradients in $z_{0}$, at this position, carrier density is maximum and electrons are shifted, this situation explain an open circuit and the junction width extend to $z_{0}$,

3. The negative gradient corresponding to $z \succ z_{0}$ where electrons are recombined in volume in the base. This decay is been explained by Beer Lambert law [19];

We also note that the maximum of carriers density increase with the magnetic field increase. This is explained by the storage of charge carriers across the junction.

This behavior of maximum of charge carriers were not only explained by the decrease of diffusion length and diffusion coefficient but also by the number of electrons which through the junction with the increase of magnetic field.

So the space charge width extension has affected by the magnetic field variations.

\subsection{Effects Magnetic Field and Air Mass on Junction Capacitance}

The solar cell junction behaves like a capacitor with capacitance $\mathrm{C}[2,20]$ depending on the space charge region width. However, the expression of junction capacity has been given by [21]:

$$
C\left(a_{m}, b_{m}, B\right)=\frac{Q\left(a_{m}, b_{m}, B\right)}{V\left(a_{m}, b_{m}, B\right)}
$$

where:

$$
Q\left(a_{m}, b_{m}, B\right)=q \int_{-a-b}^{a} \int_{-b}^{b} \delta\left(x, y, 0, a_{m}, b_{m}, B\right) d x d y
$$

with:

q: elementary charge;

-V: transient voltage;

-B: Magnetic field;

and:

-the exponential expression of transient voltage is given by (23):

$$
\begin{gathered}
V\left(a_{m}, b_{m}, B, t\right)=V_{T} F v\left(k_{1} ; l^{*} ; \mu_{1}\right) \cdot R \cdot \exp \left(-\beta^{*}(t-T e)\right) \\
R=\exp \left(\frac{\Delta V}{V_{T}}\right)
\end{gathered}
$$

Through the figure 4, junction capacitance is plotted versus magnetic field for different illumination air mass values. 


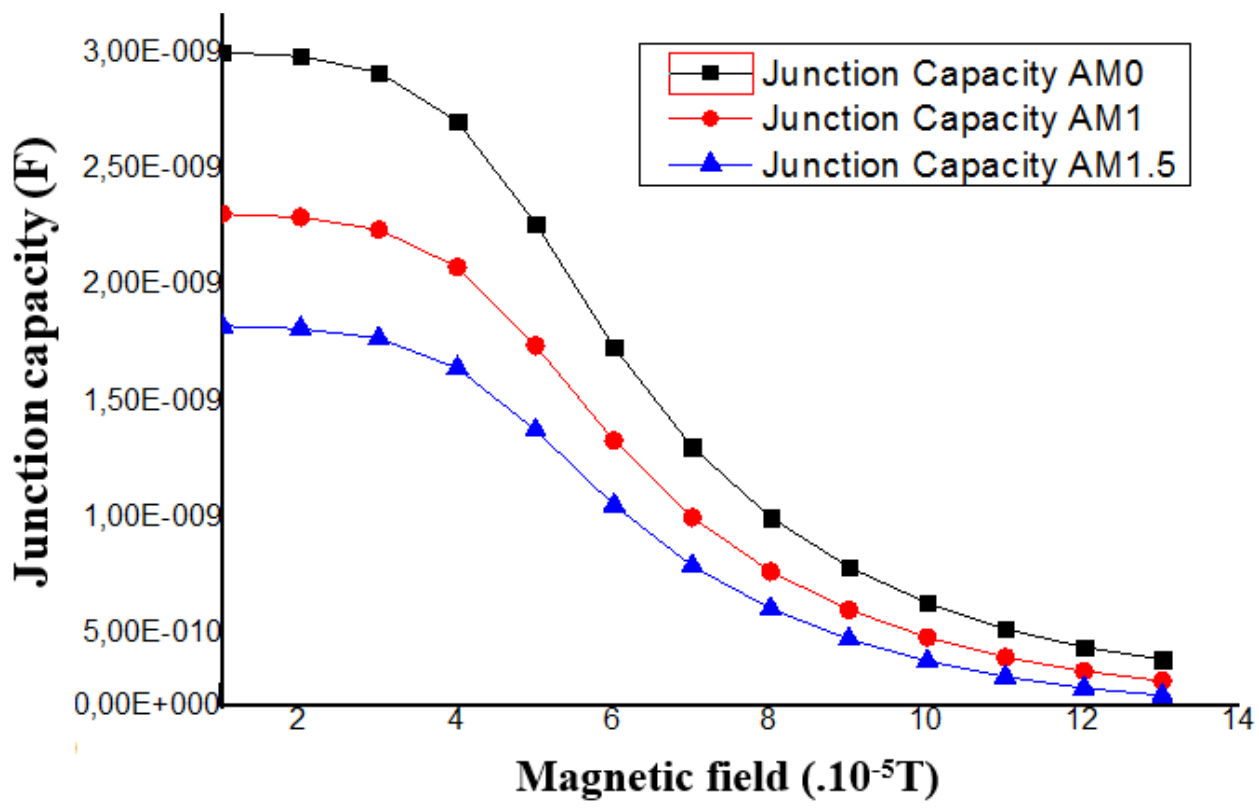

Figure 4. Junction capacitance profiles versus magnetic field for various illumination air mass. $H=200 \mu m$

The curves of figure 4 shows the decay of junction capacitance with the magnetic field but this decay is slow for small values of magnetic field. So the junction capacitance seems not to depend on magnetic field for values less than $B \leq B_{0}$ by presenting a small tray. But for more intense magnetic field $B \succ B_{0}$, the junction capacitance decreases very rapidly. This behavior of the junction capacitance is directly due to the space charge region widening with magnetic field.

Analyzing the influence of illumination air mass on the solar cell, capacitance behaviors poorly once illumination air mass is increasing, this in case of open circuit mode.

In fact increasing the illumination air mass means that intensity of light is reduced: hence the amount of charge carriers decreases also. But once in short circuit mode, capacitance is very poor and also influence of illumination air mass is slow.

\subsection{Effects of Magnetic Field and Air Mass on the Reverse of Junction Capacitance}

The reverse of junction capacitance is proportional to the space charge region width. Then we can also deduce the effects of magnetic field and air mass on the space charge region width extension.

The figure 5 present the profiles of the reverse of junction capacitance with magnetic field and air mass illumination.

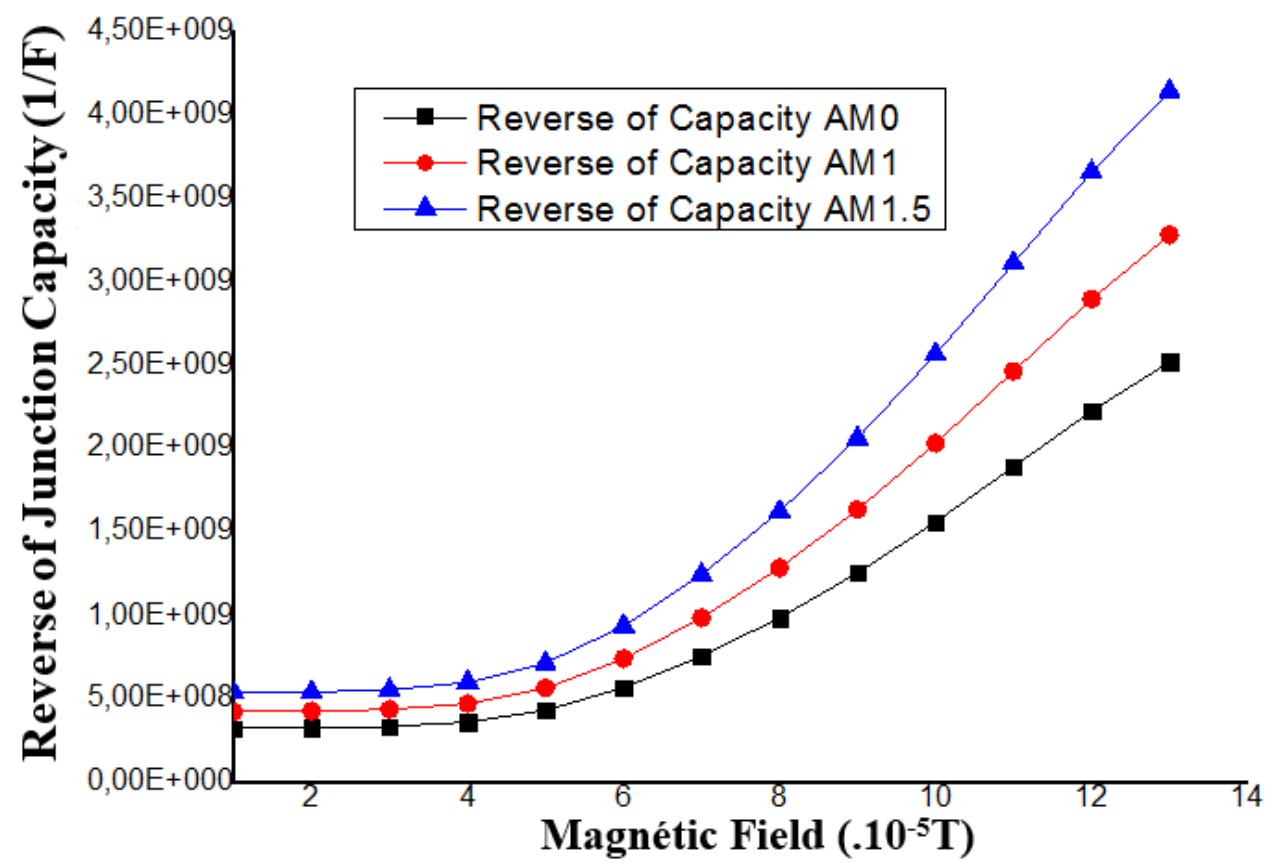

Figure 5. Reverse of junction capacitance profiles versus magnetic field with various illumination air mass. $H=200 \mu m$ 
The reverse of junction capacitance increase when magnetic field whatever the illumination air mass. So this increase is slow for $B \leq B_{0}$ but for more intense values of magnetic field $B \succ B_{0}$ this increase is faster. Then space charge width is reduced when the magnetic field increase

The decrease of illumination air mass number translate the increase of photogeneration charge carriers, so the reverse of maximum of carriers density decrease reducing the reverse of junction capacitance and the space charge region width.

The space charge region width extension is affected by the magnetic field and air mass illumination.

\section{Conclusion}

In this paper, a three dimensional approach of electrons diffusion in the $p$ region of a bifacial polycrystalline silicon solar cell is presented. The solar cell is front side illuminated by a pulsed light under external magnetic field versus various air mass illumination values.

This manuscript shows the effects of air mass illumination and magnetic field on carriers' density, junction capacitance decay and on junction capacitance reverse. The influences of magnetic field and air mass illumination on space charge region extension are pointed out.

In summary, the limiting effects for larges values of magnetic field on the quality of junction capacitance and air mass illumination impacts of a solar cell are pronounced. The air mass illumination and large values of magnetic field affect the solar cell space charge region width extension.

\section{References}

[1] Betser, Y., Ritter, D., Bahir, G., Cohen, 3D Study of Bifacial silicon solar cell under intense light Sperling, J., 1995. App. Phys. Lett. 67, (13): 1883-1884.

[2] Serafettin Erel., 2002. Solar Energy Materials \&Solar Cells, 71: 273-280.

[3] S. Madougou1, F. Made, M. S. Boukary and G. Sissoko: I -V Characteristics for Bifacial Silicon Solar Cell Studied under a Magnetic Field; Advanced Materials Research Vols. 18-19 (August 2007) pp. 303-312.

[4] Coors, S., Schneider, B and Bohm, M., 1998.2nd World conference and exhibition on photovoltaic solar energy conversion.

[5] Pelanchon, F., Sudre, C and Moreau, Y., 1992.11th European Photovoltaic Solar Energy Conference.

[6] Dieng, A., Sow, M. L., Mbodji, S., Samb, M. L., Ndiaye, M., Thiame, M., Barro, F. I and Sissoko, G., 2009. Proceedings of 24th European Photovoltaic Solar Energy Conference.
[7] M. Zoungrana, I. Zerbo, A. Sere, B. Zouma AND F. Zougmore, 3D Study of Bifacial Silicon Solar Cell Under Intense Light Concentration and Under External Constant Magnetic Field Global Journal of Engineering Research VOL 10, NO. 1\&2, 2011: 113-124.

[8] Agroui, K., Belghachi, A and et Kadri, S. Caractérisations électriques et thermiques d'un module PV au silicium multicristallin en milieu contrôle et sur site saharien. Rev. Energ. Ren.: ICPWE, 2003, pp: 19-25.

[9] Rev. Energ. Ren.: IPWE (2003) 19-25.

[10] Ala, a H. Shnishil; Influence of Air Mass on the Performance of Many Types of PV Modulus in Baghdad; 2011; (2011) 153-159.

[11] Khalid S Rida, Ali AK Al-Waeli, Kadhem AH Al-Asadi; The impact of air mass on photovoltaic panel performance doi: 10.18282/ser.v1.i1.41.

[12] Barro, F. I., Mbodji, S., Ndiaye, M., Ba, E and Sissoko, G., 2008. Proceedings of 23rd European Photovoltaic Solar Energy Conference.

[13] Ba, B., Kane, M and Sarr, J., 2003. Solar Energy Materials \& Solar Cells 80: 143-154.

[14] J. P Charles, A. Haddi, A. Maouad, H. Bakhtiar, A Zerga, A Hoffmann, P Mialhe; "La Jonction du Solaire à la Microélectronique” Rev. Energ. Ren., 3; 2000, 1-16.

[15] F. Toure, M. Zoungrana, B. Zouma, S. Mbodji, S. Gueye, A. Diao \& G. Sissoko. Influence of Magnetic Field on Electrical Model and Electrical Parameters of a Solar Cell under Intense Multispectral Illumination, Global Journal of Science Frontier Research Physics and Space Sciences Volume 12 Issue 6 Version 1.0 Year 2012.

[16] R. Sam and al; 3D determination of the minority carrier lifetime and the p-n junction recombination velocity of a polycrystalline silicon solar cell; IOP Conf. Series: Materials Science and Engineering 29 (2012).

[17] R. Sam, K. Kaboré and F. Zougmoré: A Three-Dimensional Transient Study of a Polycrystalline Silicon Solar Cell under Constant Magnetic Field; International Journal of Engineering Research Volume No. 5, Issue No. 2, pp.: 93- 97.2016.

[18] Joze et al: Approximation of the carrier generation rate in illuminated silicon; Solid State Electronics vol 28; No 12; pp. 1241-1243.1985.

[19] S. N. Mohammad; An alternative method for the performance analysis of silicon solar cells, J. Appl. Phys. 61 (2), (1987) pp 767-777.

[20] F. Touré, M. Zoungrana, R. Sam, M. T. D. Diop, F. I. Barro G. Sissoko Effet du champ magnétique sur le rendement de la capacité d'une photopile monofaoiciale au silicium par le modèle de l'extension de la zone de charge d'espace; J. Sci. Vol. $10, \mathrm{~N}^{\circ} 4(2010) 16-22$.

[21] G. Sissoko and al: Silicon solar cell space charge width determination by study in modelling ", Proc. World Renewable Energy Congress, 1998, 1852-1855. 